\title{
PRODUCTION OF PROSTAGLANDINS AND LEUKOTRIENES BY
}

\author{
Paracoccidioides brasiliensis
}

Thesis: G. A. Biondo submitted this thesis for his Masters in Tropical Diseases at the Botucatu Medical School, São Paulo State University, UNESP, Botucatu, São Paulo State, Brazil, 2008.

Advisor: Professor Angela Maria Victoriano de Campos Soares

Paracoccidioides brasiliensis is the causative agent of paracoccidioidomycosis, the most prevalent deep mycosis in Latin America. Production of eicosanoids, including prostaglandins and leukotrienes, during fungal infections is theorized to play a critical role on fungal survival and/or growth as well as on host immune response modulation. Host cells are one source of these mediators; however another potential source may be the fungus itself. The purpose of our study was to assess whether $P$. brasiliensis strains with different degree of virulence ( $\mathrm{Pb} 18$, $\mathrm{Pb} 265, \mathrm{PbBT79}, \mathrm{Pb} 192)$ produce both, prostaglandin $\mathrm{E}_{2}\left(\mathrm{PGE}_{2}\right)$ and leukotriene $\mathrm{B}_{4}$ $\left(\mathrm{LTB}_{4}\right)$. Moreover, we asked if $P$. brasiliensis can use exogenous sources of arachidonic acid (AA), as well as metabolic pathways dependent on cyclooxygenase (COX) and lipoxygenase (5-LO ) enzymes, for $\mathrm{PGE}_{2}$ and $\mathrm{LTB}_{4}$ production, respectively. Finally, a possible association between these eicosanoids and fungus viability was assessed. We demonstrated, using ELISA assays, that all $P$. brasiliensis strains, independently of their virulence, produce high $\mathrm{PGE}_{2}$ and $\mathrm{LTB}_{4}$ levels after a 4-hour culture, which were reduced after 8 hours. However, in both culture times, higher eicosanoids levels were detected when culture medium was supplemented with exogenous AA. Differently, treatment with indomethacin, a COX inhibitor, or MK886, a 5-LO inhibitor, induces a reduction on $\mathrm{PGE}_{2}$ and $\mathrm{LTB}_{4}$ levels, respectively, as well as in fungus viability. The data provide evidence that $P$. brasiliensis is able to metabolize either endogenous or exogenous AA by pathways that depend on COX and 5-LO enzymes for producing, respectively, $\mathrm{PGE}_{2}$ and LTB4 that are critical for its viability.

KEY WORDS: arachidonic acid, eicosanoids, leukotrienes, Paracoccidioides brasiliensis, prostaglandin.

CORRESPONDENCE TO:

GUILHERME AUGUSTO BIONDO, Departamento de Doenças Tropicais, Faculdade de Medicina de Botucatu, UNESP, Botucatu, SP, 18618-000, Brasil. Phone: +55 14 3882-6022. Email: quibiondo@yahoo.com.br. 